\title{
Associations between dietary insulin load with cardiovascular risk factors and inflammatory parameters in elderly men: a cross-sectional study
}

\author{
Hadis Mozaffari ${ }^{1}$, Nazli Namazi ${ }^{2}$, Bagher Larijani ${ }^{3}$, Pamela J. Surkan ${ }^{4}$ and Leila Azadbakht ${ }^{1,2,5 *}$ \\ ${ }^{1}$ Department of Community Nutrition, School of Nutritional Sciences and Dietetics, Tehran University of Medical Sciences, \\ 141556117 Tehran, Iran \\ ${ }^{2}$ Diabetes Research Center, Endocrinology and Metabolism Clinical Sciences Institute, Tebran University of Medical Sciences, \\ 1411713137 Tehran, Iran \\ ${ }^{3}$ Endocrinology and Metabolism Research Center, Endocrinology and Metabolism Clinical Sciences Institute, Tebran \\ University of Medical Sciences, 1411713137 Tehran, Iran \\ ${ }^{4}$ Department of International Health, John Hopkins Bloomberg School of Public Health, Baltimore, MD 21205, USA \\ ${ }^{5}$ Department of Community Nutrition, School of Nutrition and Food Science, Isfahan University of Medical Science, \\ 8174673461 Isfahan, Iran
}

(Submitted 4 April 2018 - Final revision received 20 November 2018 - Accepted 3 December 2018)

\section{Abstract}

Given the limited research on dietary insulin load (DIL), we examined DIL in relation to cardiovascular risk factors and inflammatory biomarkers in elderly men. For the present cross-sectional study, we recruited 357 elderly men. Dietary intake was assessed using FFQ. DIL was estimated by multiplying the insulin index of each food by its energy content and frequency of consumption and then summing the final value of all food items. After adjustment for covariates, a significant positive association was observed between high DIL with fasting blood sugar (FBS) levels (OR: 7.52; 95\% CI 3.38, 16.75; $P=0.0001$ ) and high-sensitive C-reactive protein (hs-CRP) (OR: 3.03; 95\% CI 1.54, 5.94; $P=0 \cdot 001)$. However, there was no association between high DIL and BMI (OR: $1.43 ; 95 \%$ CI $0 \cdot 75,2 \cdot 75 ; P=0 \cdot 27$ ), serum TAG level (OR: $0 \cdot 82$; $95 \%$ CI $0 \cdot 26,2 \cdot 59 ; P=0 \cdot 73$ ), HDL-cholesterol (OR: $2 \cdot 03 ; 95 \%$ CI $0 \cdot 79,5 \cdot 23 ; P=0 \cdot 13)$ and fibrinogen (OR: $1.57 ; 95 \%$ CI $0 \cdot 80,3 \cdot 06 ; P=0 \cdot 18)$. Overall, elderly men with high DIL had higher FBS and hs-CRP levels than those with low DIL. Future studies are needed to clarify the association between DIL and other cardiovascular risk factors in both men and women.

Key words: Dietary insulin load: Cardiovascular risks: Inflammation: Elderly

CVD is the most common cause of death in the world ${ }^{(1)}$. Approximately one third of deaths are related to $\mathrm{CVD}^{(1)}$. In 2010, the prevalence of CVD among individuals over 65 years old was $19.8 \%$ in the $\mathrm{USA}^{(2)}$.

Older people are at higher risk of obesity ${ }^{(3)}$. Obesity with insulin resistance is associated with hyperinsulinaemia ${ }^{(4)}$ and hyperinsulinaemia can lead to dyslipidaemia ${ }^{(5)}$, high blood pressure $^{(6)}$ and inflammation ${ }^{(7)}$. Moreover, obesity in the elderly can increase inflammatory parameters that lead to dyslipidaemia and insulin resistance ${ }^{(3)}$.

The potential use of diet to induce postprandial insulin secretion is likely to be critical for managing dyslipidaemia, weight gain and inflammation ${ }^{(8-12)}$. Although evidence has been accumulating regarding specific dietary factors and insulin resistance ${ }^{(13-15)}$, dietary indices that examine the overall dietary patterns may be more informative. Dietary insulin load (DIL) is an example of one such index ${ }^{(16)}$.
The insulin index represents the insulin response to isoenergetic components of foods in comparison to a reference food (glucose or white bread) ${ }^{(17)}$. Insulin index is based on postprandial insulin secretion that is evoked through mixed meals ${ }^{(17)}$. This index takes into account not only carbohydrate-containing foods but also high-fat, highprotein foods and their interactions ${ }^{(18)}$. Given that insulin index is based on insulin secretion, a link between insulin exposure and propensity to chronic diseases might exist $^{(11,19)}$. DIL is another dietary index that is estimated through multiplying the reported insulin index value of each food by its energy content and the frequency of consumption of each food ${ }^{(16)}$.

A Finnish study with 22 years of follow-up demonstrated that insulin was a suitable predictor of coronary disease ${ }^{(20)}$. Limited research exists on the association between insulin indices and CVD risk factors, with existing literature lacking systematic

Abbreviations: DIL, dietary insulin load; FBS, fasting blood sugar; hs-CRP, high-sensitive C-reactive protein; SES, socio-economic status.

* Corresponding author: L. Azadbakht, fax +98 2188984861, email azadbakhtleila@gmail.com 
evaluation across studies focusing on the same risk factors. Mirmiran et al. ${ }^{(16)}$ showed an inverse association between DIL and insulin resistance. Nimptsch et al. ${ }^{(11)}$ also found an inverse association between DIL and HDL-cholesterol and a positive association between DIL and TAG, particularly among obese individuals. In a prospective study by Joslowski et al. ${ }^{(21)}$, DIL was associated with body fat mass, while no relation to BMI was observed.

Elderly subjects might be more at the risk for insulin resistance due to their body compositions and metabolic profiles, ${ }^{(3)}$ calling for the examination of the association between the DIL and cardiovascular risk factors in this population. Moreover, men are at higher risk of CVD compared with women ${ }^{(22)}$. Research shows that CVD develops approximately 7-10 years earlier in men compared with women ${ }^{(23)}$. Due to the limited studies on DIL and its association with cardiovascular biomarkers, our aim was to examine the association between DIL and cardiovascular risk factors in elderly men.

\section{Methods}

\section{Study population}

To date, little attention has been paid to men, especially the elderly men, so we prioritised this group in our study. In this cross-sectional study, we used clustered random sampling to select men referred to ten health centres in southern Tehran, Iran (March to August 2017). To calculate the number of men to be sampled from each health centre, the total population served by each centre was represented proportionally in the calculated sample size ( $n$ 313). We included men over the age of 60 years who were not already adhering to specific diets. Men were excluded if they had any malignant disease such as cancer. Moreover, we excluded subjects from our analyses with under- and overreported total energy intake $(<3347$ and $>17573 \mathrm{~kJ} / \mathrm{d})^{(24)}$. High-sensitive C-reactive protein (hs-CRP) was considered the main dependent variable for calculating the sample size $\mathrm{s}^{(25)}$. For the sample size calculation, we defined $\alpha=0 \cdot 05, d=4 \%$ and the effect size $=1 \cdot 5$. Finally, based on hsCRP values, we determined that 313 individuals were needed. However, to compensate for potential exclusion of participants due to under- and overreporting of total energy intake, 365 subjects were selected for inclusion. After exclusion of participants who under- and overreported the total energy intake ( $n$ 8), 357 remained in the analysis. Therefore, under- and overreported total energy intake was the only reason for exclusion. Written informed consent was obtained from all participants. Ethical approval for this protocol was given by the National Institute for Medical Research Development (grant and ethics number: 965430).

\section{Dietary assessment}

Participants' usual dietary intake was obtained using a 168item semi-quantitative FFQ through face-to-face interviews with a trained nutritionist. The validity and reliability of this questionnaire has been previously reported to be adequate ${ }^{(26)}$. Participants were asked to report on average frequencies of their food consumption on a daily, weekly or monthly basis. The portion size of each food was translated from household measures into grams. An adapted version of NUTRITIONIST IV modified for Iranian foods (version 7.0; N-Squared Computing) was used to calculate mean energy and nutrient intakes $^{(25,27,28)}$.

Calculation of dietary insulin load. The insulin index of each food was extracted for analysis (based on methods outlined in Kirstine Bell's thesis) ${ }^{(29)}$. Insulin index was defined as the AUC representing insulin (during $120 \mathrm{~min}$ ) in response to intake of approximately a $1000 \mathrm{~kJ}$ portion of the test food, which then was divided by the area below the curve after consumption of an isoenergetic reference food ${ }^{(17)}$. The average value of DIL for each study participant during the previous year was computed using FFQ data. In this fashion, the insulin index value of each food item was multiplied by its energy content and also by the frequency of consumption. Finally, all food item values were summed. The formula used was as follows:

Insulin load of food $=$

$\sum$ (Insulin index of food

$\times$ energy content of food ( $\mathrm{kcal} /$ serving)

$\times$ frequency of consumption (serving of food/d))

\section{Biochemical assessment}

For each subject, a single venous blood sample was taken after $12 \mathrm{~h}$ of fasting. Serum concentrations for fasting blood sugar (FBS), lipid profiles including total cholesterol, LDL-cholesterol, HDL-cholesterol and TAG were quantified using commercial enzymatic reagents (Pars Azmoon). Insulin serum levels were measured using the ELISA method (ELISA; Diagnostic Biochem Canada, Inc.). hs-CRP concentrations were assessed using an ultrasensitive latex-enhanced immunoturbidimetric assay (Randox Laboratory Ltd). Serum levels of inflammatory biomarkers were determined using the ELISA method (Boster Biological Technology for IL- 6 and TNF- $\alpha$ ). We used the Clauss clotting method that involves recording the rate of fibrinogen conversion to fibrin in the presence of thrombin. Insulin resistance and insulin sensitivity were assessed using the homoeostasis model assessment for insulin resistance (HOMA-IR) ${ }^{(30)}$ and the quantitative insulin-sensitivity check index (QUICKI) ${ }^{(31)}$, respectively.

\section{Anthropometric assessment}

Anthropometric indices (body weight, height and waist circumference) were measured by a trained nutritionist. Body weight was measured using calibrated digital scales (SECA 813; Seca) after participants had removed their shoes and any heavy clothes. Body weight was reported within $100 \mathrm{~g}$ of precision. Height was measured using a tape metre (with measurement 
precision of $0.5 \mathrm{~cm}$ ), while participants were standing against a wall and their shoulders were in a normal position. Waist circumference was measured at the narrowest point between the inferior rib and iliac crest over light clothing without applying pressure to the body. It was recorded to the nearest $0.5 \mathrm{~cm}$. BMI was calculated as body weight in $\mathrm{kg}$ divided by height in $\mathrm{m}^{2}$.

\section{Assessment of other variables}

Blood pressure was measured twice while participants were in a seated position for $10 \mathrm{~min}$. Participants waited at least $30 \mathrm{~s}$ between the first and second measurements. The average of the two readings was used as the final blood pressure. Socioeconomic status (SES) was assessed using a questionnaire that has been validated and is reliable in the Iranian population and that was developed for measuring SES and its association with health outcomes ${ }^{(32)}$. A total standardised score for all participants was computed (using factor analysis and a summary index), then its compliance with a normal summary index was also examined using a Kappa test. This questionnaire consists of questions about educational level, participant job, car or house ownership, having modern appliances, number of family members and trips inside or outside the country during the last year. The reported correlation of these parameters with the total score was $0 \cdot 87$. In the current study, participant SES was described for each category of DIL based on the calculated total scores.

\section{Statistical analysis}

The Kolmogorov-Smirnov test and histogram curves were used to examine whether variables had normal distributions. Parameters with normal distributions were presented as means and standard deviations. We categorised participant characteristics, dietary intake, anthropometric indices and biochemical parameters based on the median DIL scores. Basic participant characteristics were provided for the total population and each category of DIL. To investigate the differences in characteristics between categories of DIL, $\chi^{2}$ (qualitative variables) and independent $t$ tests (quantitative variables) were used. Dietary intakes within categories of DIL were compared using ANCOVA to adjust for daily energy intake. The levels of anthropometric measures, and biochemical parameters within categories of DIL were compared using independent $t$ tests in crude models and ANCOVA in adjusted models. To assess the association between DIL and cardiometabolic risk factors, binary logistic crude and adjusted regression models were used. In the adjusted models, we controlled for a wide range of confounders (model 1: energy intake, marital status, SES and smoking; model 2: energy intake, marital status, SES, smoking, disease status, anti-diabetic drugs, thyroid drugs and heart disease drugs). The low category of DIL was considered the reference group and high and low categories were compared to predict the risk of CVD. Glycaemic control parameters and lipid profiles were considered primary outcomes, while inflammatory biomarkers were considered secondary outcomes. All statistical analyses were performed using SPSS software (version 18; SPSS Inc.). $P<0.05$ was considered statistically significant.

\section{Results}

The mean age of participants ( $n$ 357) was 64.96 years. General participant characteristics in the two DIL categories are represented in Table 1. A larger percentage of participants in the high category were married $(P=0.003)$, had lower education levels $(P=0.009)$, were non-smokers $(P=0.0001)$, had no disease $(P=0.02)$, did not use anti-diabetic drugs $(P=0 \cdot 02)$, thyroid drugs $(P=0.001)$ or drugs for heart disease $(P=0.0001)$.

Participant dietary intakes in each DIL category are represented in Table 2. Participants in the high DIL category had higher consumption of energy $(P=0.0001)$, carbohydrates $(P=0.0001)$, fruits $(P=0.002)$, vegetables $(P=0.006)$, meats $(P=0.04)$ and grains $(P=0.0001)$, compared with those in the low category. However, participants who were in the high category of DIL had lower fat $(P=0.02)$ and oil consumption $(P=0 \cdot 0001)$.

Participants' anthropometric measurements and biochemical markers are displayed in Table 3. With regard to blood pressure, participants in the high DIL category had higher systolic blood pressure $(P=0.004)$, insulin $(P=0.0001)$, HOMA-IR $(P=0.005)$ and hs-CRP $(P=0.04)$ levels compared with participants in the low category. However, the differences between anthropometric measurements, glycaemic parameters, lipid profiles, liver enzymes and inflammatory biomarkers did not significantly differ between elderly men classified in the low and high DIL categories.

OR and $95 \%$ CI for cardiovascular risk factors by medians of DIL are provided in Table 4 . In subjects who had diets with high DIL, serum levels of FBS were 7.52 times greater than those with low DIL (OR: $7.52 ; 95 \%$ CI 3.38, 16.75; $P=0.0001$ ). Moreover, subjects with high DIL showed 3.03 times greater hsCRP levels than those with low DIL (OR: 3.03; 95\% CI 1.54, 5.94; $P=0.001)$. No associations were found between high DIL and BMI (OR: $1.43 ; 95 \%$ CI $0 \cdot 75,2 \cdot 75 ; P=0.27$ ), serum levels of TAG (OR: $0 \cdot 82 ; 95 \%$ CI 0.26, 2.59; $P=0 \cdot 73$ ), HDL-cholesterol (OR: $2 \cdot 03$; $95 \%$ CI $0 \cdot 79,5 \cdot 23 ; P=0 \cdot 13$ ) or fibrinogen (OR: 1.57 ; $95 \%$ CI $0 \cdot 80,3 \cdot 06 ; P=0 \cdot 18)$.

\section{Discussion}

In the present cross-sectional study, DIL was positively associated with serum levels of FBS and hs-CRP. However, there was no association between DIL and BMI or between DIL and lipid profiles. To the best of our knowledge, this is the first study, in which glycaemic parameters, lipid profile and also inflammatory biomarkers were investigated to provide better insight into the association between DIL and CVD risk factors in elderly men.

DIL is an indicator that adequately reflects insulin secretion of the whole diet, rather than a single nutrient ${ }^{(18)}$. In the field of nutritional epidemiology, DIL is a suitable indicator to examine the link between insulin exposure and the development of metabolic diseases ${ }^{(11,19)}$. Apart from carbohydrates, dietary protein and fat can affect insulin secretion $^{(33-35)}$. Therefore, macronutrients might act synergistically to increase insulin secretion and reduce blood glucose levels ${ }^{(33-36)}$. White bread, potato, skim milk, low-fat ice 
Table 1. General participant characteristics and median dietary insulin loads (DIL) (Numbers and percentages; mean values and standard deviations)

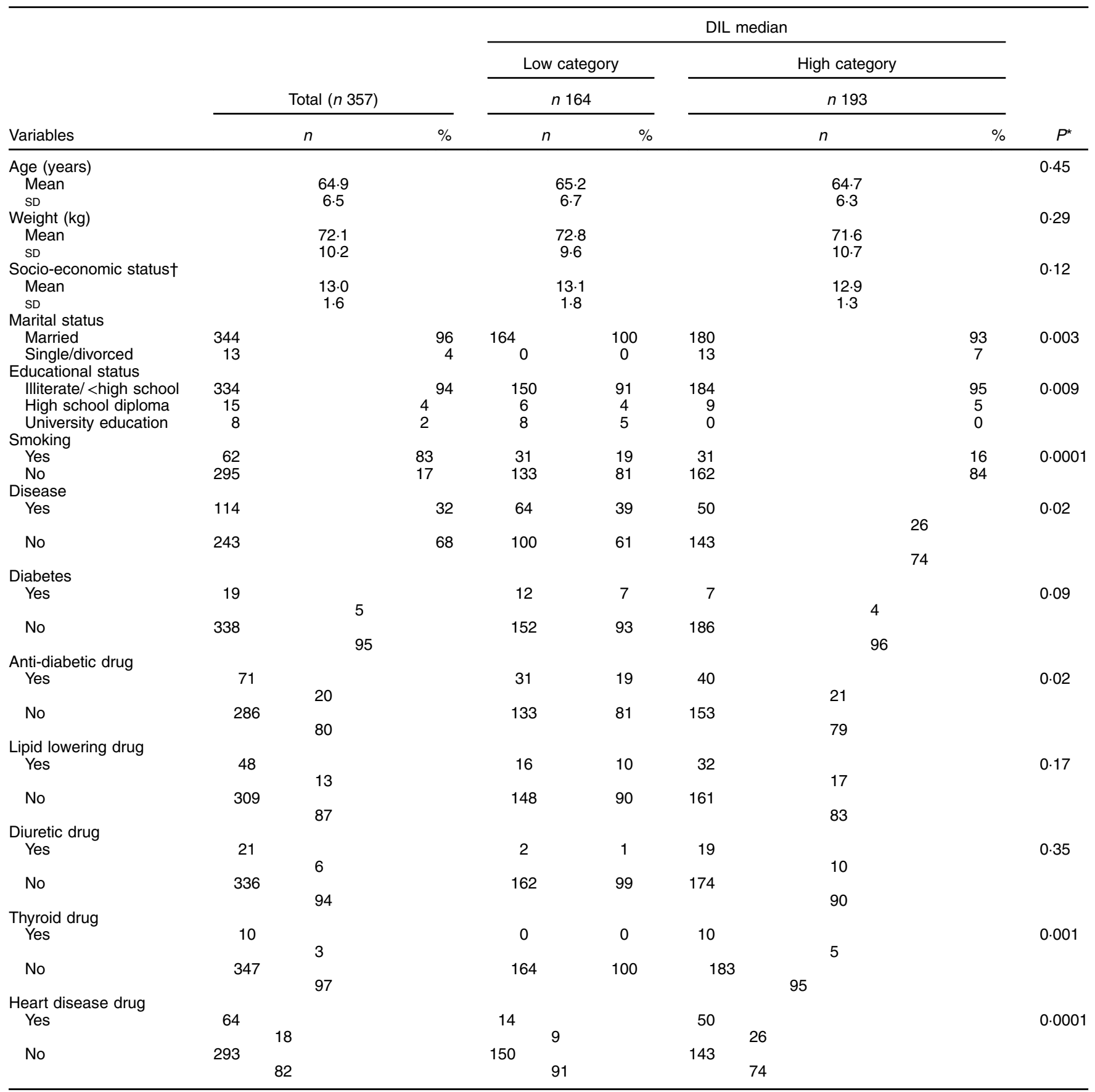

* Calculated using $x^{2}$ tests and $t$ tests for qualitative and quantitative variables, respectively.

† Socio-economic status; minimum: 10, maximum: 18.

cream or yogurt, melon, fruit juice, canned fruits, jam, chocolate and jelly beans are examples of food items with high insulin index ${ }^{(29)}$.

Associations between dietary insulin indices and metabolic features such as glycaemic status, lipid profile, inflammatory biomarkers and body composition have been addressed only in limited studies and results have been inconsistent ${ }^{(11,16,21)}$. Moreover, documented associations between diet and disease in young adults cannot be generalised to the elderly due to the differences in the grade of systematic inflammation as well as differences in the quantity and distribution of fat mass ${ }^{(3)}$. In addition, men are at higher risk of CVD compared with women ${ }^{(22)}$. Research suggests that CVD develops approximately 7-10 years earlier in men $v$. women ${ }^{(23)}$.

In the present study, DIL was not associated with BMI. These findings are consistent with a prospective study conducted by Joslowski et al. ${ }^{(21)}$. This study found that high intake of dietary insulin index (DII) (45 compared with 39) or DIL (362 
Table 2. Energy-adjusted dietary intakes and medians of dietary insulin load (DIL)

(Mean values and standard deviations)

\begin{tabular}{|c|c|c|c|c|c|}
\hline \multirow[b]{4}{*}{ Variables } & \multicolumn{4}{|c|}{ DIL median* } & \multirow[b]{4}{*}{$P+$} \\
\hline & \multicolumn{2}{|c|}{ Low category } & \multicolumn{2}{|c|}{ High category } & \\
\hline & \multicolumn{2}{|c|}{$n 164$} & \multicolumn{2}{|c|}{$n 193$} & \\
\hline & Mean & $\mathrm{SD}$ & Mean & $\mathrm{SD}$ & \\
\hline Energy (kJ/d) & 7130 & 1347 & 10615 & 2167 & 0.0001 \\
\hline Protein (g/d) & 83.74 & $19 \cdot 32$ & 82.51 & 20.97 & 0.56 \\
\hline Fat $(g / d)$ & $62 \cdot 91$ & $15 \cdot 48$ & 58.95 & $16 \cdot 80$ & 0.02 \\
\hline Carbohydrate (g/d) & $326 \cdot 26$ & $39 \cdot 16$ & 343.98 & $42 \cdot 50$ & 0.0001 \\
\hline Cholesterol (mg/d) & $182 \cdot 67$ & 88.96 & $188 \cdot 28$ & 96.53 & 0.57 \\
\hline SFA (mg/d) & $16 \cdot 52$ & $5 \cdot 88$ & $17 \cdot 22$ & $6 \cdot 38$ & $0 \cdot 29$ \\
\hline MUFA (mg/d) & $18 \cdot 04$ & $6 \cdot 27$ & $17 \cdot 98$ & $6 \cdot 80$ & 0.92 \\
\hline PUFA (mg/d) & $13 \cdot 39$ & 3.45 & $12 \cdot 27$ & $3 \cdot 75$ & 0.005 \\
\hline Fibre $(g / d)$ & $5 \cdot 91$ & 2.56 & 6.48 & 2.50 & 0.06 \\
\hline Vitamin $B_{9}(\mu \mathrm{g} / \mathrm{d})$ & 403.58 & $98 \cdot 43$ & 382.45 & $106 \cdot 81$ & 0.05 \\
\hline Vitamin $B_{1}(\mathrm{mg} / \mathrm{d})$ & 1.59 & 0.25 & 1.44 & 0.27 & 0.0001 \\
\hline Vitamin $B_{6}(\mathrm{mg} / \mathrm{d})$ & 1.89 & 0.38 & 1.82 & 0.41 & 0.13 \\
\hline Vitamin C (mg/d) & 214.03 & 83.84 & $227 \cdot 34$ & $90 \cdot 97$ & $0 \cdot 15$ \\
\hline Vitamin A (RAE/d) & $1342 \cdot 31$ & $510 \cdot 84$ & $1412 \cdot 61$ & $554 \cdot 34$ & $0 \cdot 21$ \\
\hline $\mathrm{Ca}(\mathrm{mg} / \mathrm{d})$ & $1539 \cdot 90$ & 635.90 & 1465.46 & $690 \cdot 05$ & 0.29 \\
\hline $\mathrm{Mg}(\mathrm{mg} / \mathrm{d})$ & $318 \cdot 38$ & $72 \cdot 83$ & $320 \cdot 52$ & 70.03 & 0.79 \\
\hline $\mathrm{K}(\mathrm{mg} / \mathrm{d})$ & $3957 \cdot 62$ & 933.76 & $4170 \cdot 79$ & $1013 \cdot 27$ & 0.04 \\
\hline $\mathrm{Zn}(\mathrm{mg} / \mathrm{d})$ & 9.48 & $3 \cdot 20$ & 8.91 & 3.74 & 0.11 \\
\hline $\mathrm{Fe}(\mathrm{mg} / \mathrm{d})$ & $13 \cdot 19$ & 2.94 & 11.66 & $3 \cdot 19$ & 0.0001 \\
\hline Fruit (g/d) & 357.97 & $179 \cdot 58$ & $426 \cdot 89$ & $175 \cdot 70$ & 0.002 \\
\hline Vegetables (g/d) & 359.97 & 201.98 & $427 \cdot 17$ & $197 \cdot 65$ & 0.006 \\
\hline Meat $(g / d)$ & 70.93 & $41 \cdot 85$ & $80 \cdot 96$ & $40 \cdot 97$ & 0.04 \\
\hline Grain (g/d) & $314 \cdot 22$ & $163 \cdot 20$ & 411.76 & $159 \cdot 73$ & 0.0001 \\
\hline Dairy products $(g / d)$ & 612.57 & 401.66 & $643 \cdot 82$ & 393.08 & 0.51 \\
\hline Oil (g/d) & $65 \cdot 28$ & 41.08 & $35 \cdot 32$ & $40 \cdot 28$ & 0.0001 \\
\hline
\end{tabular}

RAE, retinol activity equivalents.

${ }^{*}$ All the variables, except energy, were adjusted for energy intake.

$\dagger$ Calculated using multivariate ANCOVA.

compared with 321) during puberty (among healthy subjects) was not associated with BMI in young adulthood ${ }^{(21)}$. However, Chaput et al. ${ }^{(37)}$ showed that high insulin secretion can predict weight gain in adulthood. In Chaput et al.'s study, adults with the highest level of insulin concentration and with the lowest level of dietary fat gained approximately $4.5 \mathrm{~kg}$ more weight after 6 years of follow-up compared with those with the lowest levels of insulin and dietary fat ${ }^{(37)}$. It has been demonstrated that high insulin secretion due to high consumption of insulinogenic foods during a long period can result in the development of fat mass ${ }^{(21)}$ and insulin resistance ${ }^{(16)}$. Following insulin resistance, the risk of obesity can increase ${ }^{(38)}$. Moreover, high insulin concentrations can suppress lipolysis and stimulate glucose uptake, which in turn enhances lipogenesis in adipocytes $^{(39)}$.

Regarding glycaemic control, a significant positive association was observed between DIL and FBS concentrations. Although high secretion of insulin can result in lower FBS levels, it seems that prolonged consumption of foods with high insulin index causes $\beta$-cell dysfunction ${ }^{(11)}$. This condition subsequently can lead to insulin resistance and increased serum glucose levels.

We found no association between DIL and HDL-cholesterol concentrations. In the study by Nimptsch et al., they observed an inverse association between DIL ( $\geq 858$ compared with <648) and HDL-cholesterol. However, after stratification by
BMI, DIL was no longer associated with HDL-cholesterol levels in normal $\left(\mathrm{BMI}<25 \mathrm{~kg} / \mathrm{m}^{2}\right)$ and overweight $(\mathrm{BMI}=25-$ $29.9 \mathrm{~kg} / \mathrm{m}^{2}$ ) subjects. However, an inverse association remained among obese (BMI $\geq 30 \mathrm{~kg} / \mathrm{m}^{2}$ ) subjects ${ }^{(11)}$. A reason why we failed to observe any association between DIL and HDLcholesterol might be due to the overall low mean BMI of our participants (approximately $25.4 \mathrm{~kg} / \mathrm{m}^{2}$ ).

It appears that the inverse association between DIL and HDLcholesterol found by Nimptsch et al., especially in obese subjects, is due to the high insulin resistance in this group. A possible mechanism is that an insulinogenic diet aggravates insulin secretion, which in turn may lead to insulin resistance in the longterm, as was observed in the study by Mirmiran et al. ${ }^{(16)}$ (DIL $\geq 1097$ compared with $<794$ was associated with a $69 \%$ increase in the risk of insulin resistance). Based on previous research, insulin resistance and disturbance of glycaemic control is associated with lower HDL-cholesterol serum levels ${ }^{(40)}$. Moreover, studies have revealed that high carbohydrate consumption is associated with low serum levels of HDL-cholesterol ${ }^{(41,42)}$. In the present study, no association was found between DIL and serum TAG levels. However, in a study conducted by Nimptsch et al. ${ }^{(11)}$, a significant positive association between dietary insulin indices (DII: $\geq 46 \cdot 2$ compared with $<38 \cdot 3$; DIL: $\geq 858$ compared with $<648$ ) and TAG concentration was observed in all BMI categories, particularly in the obese. 
Table 3. Medians of dietary insulin load (DIL) by anthropometric indices, biochemical markers and blood pressure (Mean values and standard deviations)

\begin{tabular}{|c|c|c|c|c|c|}
\hline \multirow[b]{4}{*}{ Variables } & \multicolumn{4}{|c|}{ DIL median } & \multirow[b]{4}{*}{$P^{\star}$} \\
\hline & \multicolumn{2}{|c|}{ Low category } & \multicolumn{2}{|c|}{ High category } & \\
\hline & \multicolumn{2}{|c|}{$n 164$} & \multicolumn{2}{|c|}{$n 193$} & \\
\hline & Mean & SD & Mean & SD & \\
\hline \multicolumn{6}{|l|}{ BMI $\left(\mathrm{kg} / \mathrm{m}^{2}\right)$} \\
\hline Crudet & 24.83 & $2 \cdot 72$ & $26 \cdot 10$ & $3 \cdot 30$ & 0.001 \\
\hline Model $1 \ddagger$ & $25 \cdot 70$ & 3.50 & 25.06 & 3.61 & 0.16 \\
\hline Model $2 \S$ & 25.49 & 3.50 & $25 \cdot 24$ & $3 \cdot 61$ & 0.57 \\
\hline \multicolumn{6}{|l|}{$W C(\mathrm{~cm})$} \\
\hline Crude $†$ & 96.64 & 7.57 & $95 \cdot 71$ & $9 \cdot 34$ & 0.30 \\
\hline Model $1 \ddagger$ & $96 \cdot 76$ & $9 \cdot 78$ & 95.61 & $10 \cdot 13$ & 0.36 \\
\hline Model $2 \S$ & $96 \cdot 26$ & 9.78 & 96.04 & $10 \cdot 13$ & 0.86 \\
\hline \multicolumn{6}{|l|}{ SBP $(\mathrm{mmHg})$} \\
\hline Crude† & $120 \cdot 64$ & $10 \cdot 42$ & $130 \cdot 10$ & $10 \cdot 76$ & 0.008 \\
\hline Model $1 \ddagger$ & 120.58 & $10 \cdot 69$ & $130 \cdot 15$ & $10 \cdot 80$ & 0.01 \\
\hline \multirow{2}{*}{\multicolumn{6}{|c|}{ DBP $(\mathrm{mmHg})$}} \\
\hline & & & & & \\
\hline Crude† & $70 \cdot 70$ & 8.50 & 70.90 & $7 \cdot 20$ & 0.01 \\
\hline Model $1 \mp$ & $70 \cdot 78$ & 8.40 & 70.83 & $8 \cdot 30$ & 0.63 \\
\hline Model $2 \S$ & $70 \cdot 80$ & 8.40 & $70 \cdot 81$ & $8 \cdot 30$ & 0.92 \\
\hline \multicolumn{6}{|c|}{ Insulin (pmol/l) } \\
\hline Crude† & 58.33 & 28.95 & 64.93 & 38.91 & 0.07 \\
\hline Model $1 \neq$ & 54.79 & 39.37 & 67.77 & 41.45 & 0.01 \\
\hline \multirow{2}{*}{\multicolumn{6}{|c|}{ HOMA-IR }} \\
\hline & & & & & \\
\hline Crude $†$ & $2 \cdot 32$ & 1.25 & $2 \cdot 37$ & 1.67 & 0.75 \\
\hline Model $1 \neq$ & $2 \cdot 14$ & 1.69 & 2.51 & 1.25 & 0.09 \\
\hline \multirow{2}{*}{\multicolumn{6}{|c|}{ QUICKI }} \\
\hline & & & & & \\
\hline Crude $†$ & 0.34 & 0.03 & 0.35 & 0.03 & 0.30 \\
\hline Model $1 \ddagger$ & 0.35 & 0.05 & 0.35 & 0.04 & 0.91 \\
\hline Model $2 \S$ & 0.35 & 0.03 & 0.34 & 0.04 & 0.21 \\
\hline TAG (mmol//) & & & & & \\
\hline Crude† & 1.47 & 0.36 & 1.47 & 0.47 & 0.94 \\
\hline Model $1 \ddagger$ & 1.50 & 0.49 & 1.44 & 0.51 & 0.30 \\
\hline Model $2 \S$ & 1.49 & 0.49 & 1.45 & 0.51 & 0.49 \\
\hline $\mathrm{HDL}-\mathrm{C}(\mathrm{mmc}$ & & & & & \\
\hline Crude† & 1.24 & 0.21 & 1.30 & 0.23 & 0.01 \\
\hline Model $1 \ddagger$ & $1 \cdot 30$ & 0.24 & 1.25 & 0.26 & 0.12 \\
\hline Model $2 \S$ & 1.29 & 0.23 & 1.25 & 0.24 & 0.18 \\
\hline LDL-C (mmo & & & & & \\
\hline Crude† & $2 \cdot 39$ & 0.55 & $2 \cdot 51$ & 0.53 & 0.03 \\
\hline Model 1f & $2 \cdot 44$ & 0.60 & $2 \cdot 46$ & 0.63 & 0.83 \\
\hline Model $2 \S$ & $2 \cdot 44$ & 0.57 & 2.46 & 0.59 & 0.71 \\
\hline TC $(\mathrm{mmol} / \mathrm{l})$ & & & & & \\
\hline Crude† & 4.43 & 0.70 & $4 \cdot 68$ & 0.59 & 0.0001 \\
\hline Model $1 \ddagger$ & 4.49 & 0.68 & 4.62 & 0.71 & 0.13 \\
\hline Model $2 \S$ & 4.50 & 0.60 & $4 \cdot 61$ & 0.63 & 0.14 \\
\hline hs-CRP $(\mu \mathrm{g} / \mathrm{r}$ & & & & & \\
\hline Crudet & 1.65 & 0.87 & 1.94 & 0.88 & 0.002 \\
\hline Model $1 \ddagger$ & 1.75 & 0.96 & 1.86 & 0.97 & 0.39 \\
\hline Model $2 \S$ & 1.67 & 0.96 & 1.92 & 0.97 & 0.04 \\
\hline Fibrinogen $(\mathrm{g}$ & & & & & \\
\hline Crude† & $2 \cdot 82$ & 0.52 & $2 \cdot 67$ & 0.40 & 0.002 \\
\hline Model $1 \ddagger$ & $2 \cdot 75$ & 0.53 & $2 \cdot 74$ & 0.55 & 0.86 \\
\hline Model $2 \S$ & $2 \cdot 73$ & 0.53 & $2 \cdot 75$ & 0.55 & 0.84 \\
\hline IL-6 (pg/ml) & & & & & \\
\hline Crude† & $6 \cdot 49$ & 0.74 & $6 \cdot 46$ & 0.74 & 0.75 \\
\hline Model $1 \ddagger$ & $6 \cdot 45$ & 0.84 & $6 \cdot 49$ & 0.83 & 0.70 \\
\hline Model $2 \S$ & 6.45 & 0.84 & 6.49 & 0.83 & 0.70 \\
\hline TNF- $a(\mathrm{pg} / \mathrm{m}$ & & & & & \\
\hline Crudet & 0.72 & 0.08 & 0.72 & 0.07 & 0.78 \\
\hline Model 1f & 0.72 & 0.09 & 0.72 & 0.09 & 0.73 \\
\hline Model $2 \S$ & 0.72 & 0.09 & 0.72 & 0.09 & 0.75 \\
\hline
\end{tabular}

WC, waist circumference; SBP, systolic blood pressure; DBP, diastolic blood pressure; HOMA-IR, homoeostasis model assessment-insulin resistance; QUICKI, quantitative insulin sensitivity check index; TC, total cholesterol; hs-CRP, high-sensitive C-reactive protein.

* Calculated using $t$ tests for the crude model and ANCOVA in the adjusted models.

$\dagger$ Crude: not adjusted for any variables.

† Model 1: this model was adjusted for energy intake, marital status (which includes educational level), socio-economic status and smoking

$\S$ Model 2: this model was adjusted for energy intake, marital status, socio-economic status (which includes educational level), smoking, disease, anti-diabetic drugs, thyroid drugs and heart disease drugs. 
Table 4. Crude and multivariable OR and $95 \% \mathrm{Cl}$ in medians of dietary insulin load (DIL) (Odds ratios and $95 \%$ confidence intervals)

\begin{tabular}{|c|c|c|c|c|}
\hline \multirow[b]{4}{*}{ Variables } & \multicolumn{3}{|c|}{ DIL median } & \multirow[b]{4}{*}{$P^{\star}$} \\
\hline & \multirow{3}{*}{$\frac{\text { Low category }}{n 164}$} & \multicolumn{2}{|c|}{ High category } & \\
\hline & & \multicolumn{2}{|c|}{$n 193$} & \\
\hline & & OR & $95 \% \mathrm{Cl}$ & \\
\hline \multicolumn{5}{|c|}{ Overweight and obese $\left(\mathrm{BMl}>25 \mathrm{~g} / \mathrm{m}^{2}\right)$} \\
\hline Crude & 1 & 0.88 & $0.58,1.34$ & 0.56 \\
\hline Model $1 \ddagger$ & 1 & 1.64 & $0.88,3.06$ & 0.11 \\
\hline Model $2 \S$ & 1 & 1.43 & $0.75,2.75$ & 0.27 \\
\hline \multicolumn{5}{|c|}{ FBS $(>5.55 \mathrm{mmol} / \mathrm{l})$} \\
\hline Crude & 1 & 4.64 & $2 \cdot 90,7.44$ & 0.0001 \\
\hline Model $1 \ddagger$ & 1 & 5.69 & $2.78,11.63$ & 0.0001 \\
\hline Model $2 \S$ & 1 & 7.52 & $3.38,16 \cdot 75$ & 0.0001 \\
\hline \multicolumn{5}{|c|}{ TAG $(>1.69 \mathrm{mmol} / \mathrm{l})$} \\
\hline Crude† & 1 & 1.78 & $0.80,3.92$ & 0.15 \\
\hline Model $1 \ddagger$ & 1 & 0.72 & $0.23,2.22$ & 0.57 \\
\hline Model $2 \S$ & 1 & 0.82 & $0.26,2.59$ & 0.73 \\
\hline \multicolumn{5}{|c|}{ HDL-cholesterol $(<1.03 \mathrm{mmol} / \mathrm{l})$} \\
\hline Crude† & 1 & 0.55 & $0.30,0.99$ & 0.04 \\
\hline Model $1 \ddagger$ & 1 & $2 \cdot 02$ & $0.85,4.77$ & $0 \cdot 10$ \\
\hline Model $2 \S$ & 1 & 2.03 & $0.79,5 \cdot 23$ & 0.13 \\
\hline \multicolumn{5}{|c|}{ hs-CRP (>2 mg/l) } \\
\hline Crude† & 1 & 1.92 & $1 \cdot 25,2 \cdot 93$ & 0.003 \\
\hline Model 1f & 1 & 1.97 & $1.08,3.59$ & 0.02 \\
\hline Model $2 \S$ & & 3.03 & $1.54,5.94$ & 0.001 \\
\hline \multicolumn{5}{|c|}{ Fibrinogen (>2.85 g/l) } \\
\hline Crudet & 1 & 0.54 & $0.35,0.83$ & 0.005 \\
\hline Model $1 \ddagger$ & 1 & 1.43 & $0.76,2.69$ & 0.26 \\
\hline Model $2 \S$ & 1 & 1.57 & $0.80,3.06$ & 0.18 \\
\hline
\end{tabular}

FBS, fasting blood sugar; hs-CRP, high-sensitive C-reactive protein.

* Calculated using logistic regression.

$\dagger$ Crude: not adjusted for any variables.

† Model 1: the model was adjusted for energy intake, marital status, socio-economic status (which includes educational level) and smoking.

$\S$ Model 2: the model was adjusted for energy intake, marital status, socio-economic status (which includes educational level), smoking, disease, anti-diabetic drugs, thyroid drugs and heart disease drugs.

In the present study, we did not find an association between DIL and fibrinogen levels. However, there was a positive relationship between high DIL and serum levels of hs-CRP. In contrast to our study, Nimptsch et al. failed to find any association between dietary insulin indices (DII: $\geq 46 \cdot 2$ compared with <38.3; DIL: $\geq 858$ compared with $<648$ ) and inflammatory biomarkers including IL-6 and C-reactive protein (CRP). In another study, hyperglycaemia was associated with increased levels of inflammatory biomarkers (OR for CRP: 1.33; for IL-6: 1.51 and TNF- $\alpha: 1 \cdot 14)^{(43)}$. Under normal conditions, the pro-inflammatory effects of glucose are controlled by the antiinflammatory action of insulin ${ }^{(44)}$. However, in the current study, high levels of FBS in participants with high DIL (who might have reduced insulin secretion due to older age), might be an explanation for increased levels of hs-CRP.

Our failure to find relationships with a number of biomarkers may be due to several limitations. The cross-sectional design of the study prevents us from making causal inferences. Therefore, prospective studies are needed to evaluate these associations over longer periods. Second, since our study only included men, the results are not generalisable to the both sexes. Third, in this study basal insulin secretion was assessed by taking fasting insulin samples. However, DII is based on postprandial insulin secretion. Fourth, in addition to dietary factors that affect the insulin levels, it is important to consider multiple other factors that determine insulin levels such as physical activity ${ }^{(45,46)}$, anthropometric characteristics and genetic predisposition $^{(47-49)}$. Fifth, the insulin index values for foods were derived from a study that was conducted in young lean students whose insulin responses are relatively different from elderly and obese subjects ${ }^{(17)}$. However, according to a validation study, the positive link between insulin index and TAG concentrations is expected to be stronger among overweight subjects ${ }^{(11)}$. This suggests that the insulin index would also be applicable in heavier subjects. Sixth, using an FFQ as a retrospective dietary assessment tool might cause misclassification. Despite our best effort to control for major confounders, some additional confounders may not have been accounted for or residual confounding may remain. One such confounder might be recent changes in body weight as it has been shown to be associated with CVD risk factors ${ }^{(50-52)}$, particularly incidence and remission of insulin resistance ${ }^{(51)}$.

The current study has several strengths. First, limited research is available on the association between insulin indices and cardiovascular risk factors. Second, not all published studies have comprehensively taken into account different cardiovascular risk factors. However, in the present study, glycaemic parameters, lipid profile and also inflammatory biomarkers 
were investigated to provide better insight into the association between DIL and CVD risk factors. Third, as little information is available about dietary patterns and indices such as DIL in the elderly, attention to this group is critical. Fourth, the elderly are at higher risk of insulin resistance, therefore examining the association between dietary insulin indices and cardiovascular risk factors is important.

\section{Conclusion}

In this cross-sectional study, DIL was positively associated with serum FBS and hs-CRP levels. However, no association was observed between DIL and BMI or lipid profiles. More research is needed to elucidate the association between DIL and other cardiovascular risk factors and to understand potential differences by sex.

\section{Acknowledgements}

The authors thank the subjects who participated in the study.

This study was funded by the National Institute for Medical Research Development (grant no. 965430). The National Institute for Medical Research had no role in the design, analysis or writing of this article.

H. M., N. N. and L. A. designed the study. H. M., N. N., B. L., P. J. S. and L. A. contributed to the statistical analysis, interpretation of the data and to the drafting of the manuscript. The final version of the manuscript was approved by all authors before submission.

The authors declare that there are no conflicts of interest.

\section{References}

1. Pagidipati NJ \& Gaziano TA (2013) Estimating deaths from cardiovascular disease: a review of global methodologies of mortality measurement. Circulation 127, 749-756.

2. Centers for Disease Control and Prevention (2011) Prevalence of coronary heart disease-United States, 2006-2010. MMWR Morb Mortal Wkly Rep 60, 1377-1381.

3. Guarner V \& Rubio-Ruiz ME (2015) Low-grade systemic inflammation connects aging, metabolic syndrome and cardiovascular disease. Interdiscip Top Gerontol 40, 99-106.

4. Nagasaki K, Hara H, Ogawa J, et al. (1986) Relationship between hyperinsulinemia and risk factors of atherosclerosis. Ipn J Med 25, 270-277.

5. Haffner SM, Fong D, Hazuda HP, et al. (1988) Hyperinsulinemia, upper body adiposity, and cardiovascular risk factors in non-diabetics. Metabolism 37, 338-345.

6. Fournier AM, Gadia MT, Kubrusly DB, et al. (1986) Blood pressure, insulin, and glycemia in nondiabetic subjects. $\mathrm{Am} \mathrm{J}$ Med 80, 861-864.

7. Dandona P, Aljada A \& Bandyopadhyay A (2004) Inflammation: the link between insulin resistance, obesity and diabetes. Trends Immunol 25, 4-7.

8. Hensrud DD (2004) Diet and obesity. Curr Opin Gastroenterol 20, 119-124.

9. Galland L (2010) Diet and inflammation. Nutr Clin Pract 25 634-640.

10. Mekki K, Bouzidi-bekada N, Kaddous A, et al. (2010) Mediterranean diet improves dyslipidemia and biomarkers in chronic renal failure patients. Food Funct 1, 110-115.
11. Nimptsch K, Brand-Miller JC, Franz M, et al. (2011) Dietary insulin index and insulin load in relation to biomarkers of glycemic control, plasma lipids, and inflammation markers. Am J Clin Nutr 94, 182-190.

12. Setorki M, Nazari B, Asgary S, et al. (2011) Anti atherosclerotic effects of verjuice on hypocholesterolemic rabbits. Afr J Pharm Pharmacol 5, 1038-1045.

13. Pereira MA, Jacobs DR Jr, Pins JJ, et al. (2002) Effect of whole grains on insulin sensitivity in overweight hyperinsulinemic adults. Am J Clin Nutr 75, 848-855.

14. Pereira MA, Jacobs DR Jr, Van Horn L, et al. (2002) Dairy consumption, obesity, and the insulin resistance syndrome in young adults: the CARDIA Study. JAMA 287, 2081-2089.

15. Zhang Z-F, Lu J, Zheng Y-L, et al. (2013) Purple sweet potato color attenuates hepatic insulin resistance via blocking oxidative stress and endoplasmic reticulum stress in high-fat-diettreated mice. J Nutr Biochem 24, 1008-1018.

16. Mirmiran P, Esfandiari S, Bahadoran Z, et al. (2015) Dietary insulin load and insulin index are associated with the risk of insulin resistance: a prospective approach in Tehran Lipid and Glucose Study. J. Diabetes Metab Disord 15, 23.

17. Holt S, Miller J \& Petocz P (1997) An insulin index of foods: the insulin demand generated by $1000-\mathrm{kJ}$ portions of common foods. Am J Clin Nutr 66, 1264-1276.

18. Bao J, de Jong V, Atkinson F, et al. (2009) Food insulin index: physiologic basis for predicting insulin demand evoked by composite meals. Am J Clin Nutr 90, 986-992.

19. Bao Y, Nimptsch K, Meyerhardt J, et al. (2010) Dietary insulin load, dietary insulin index, and colorectal cancer. Cancer Epidemiol Biomarkers Prev 19, 3020-3026.

20. Pyörälä K, Savolainen E, Kaukola S, et al. (1985) Plasma insulin as coronary heart disease risk factor: relationship to other risk factors and predictive value during 91/2-year follow-up of the Helsinki policemen study population. J Intern Med 218, 38-52.

21. Joslowski G, Goletzke J, Cheng G, et al. (2012) Prospective associations of dietary insulin demand, glycemic index, and glycemic load during puberty with body composition in young adulthood. Int J Obes 36, 1463-1471.

22. Weidner G (2000) Why do men get more heart disease than women? An international perspective. J Am Coll Health $\mathbf{4 8}$, 291-294.

23. Maas A \& Appelman Y (2010) Gender differences in coronary heart disease. Neth Heart J 18, 598-603.

24. Saraf-Bank S, Haghighatdoost F, Esmaillzadeh A, et al. (2017) Adherence to healthy eating index-2010 is inversely associated with metabolic syndrome and its features among Iranian adult women. Eur J Clin Nutr 71, 425-430.

25. Fard NRP, Karimi M, Baghaei MH, et al. (2015) Dairy consumption, cardiovascular risk factors and inflammation in elderly subjects. ARYA Atheroscler 11, 323-331.

26. Mirmiran P, Esfahani FH, Mehrabi Y, et al. (2010) Reliability and relative validity of an FFQ for nutrients in the Tehran Lipid and Glucose Study. Public Health Nutr 13, 654-662.

27. Azadbakht L, Kimiagar M, Mehrabi Y, et al. (2007) Dietary soya intake alters plasma antioxidant status and lipid peroxidation in postmenopausal women with the metabolic syndrome. Br J Nutr 98, 807-813.

28. Esmaillzadeh A \& Azadbakht L (2011) Different kinds of vegetable oils in relation to individual cardiovascular risk factors among Iranian women. Br J Nutr 105, 919-927.

29. Bell K (2014) Clinical Application of the Food Insulin Index to Diabetes Mellitus. PhD thesis, University of Sydney, School of Molecular and Microbial Bioscience. http://hdl.handle.net/ 2123/11945 (accessed February 2019). 
30. Matthews D, Hosker J, Rudenski A, et al. (1985) Homeostasis model assessment: insulin resistance and $\beta$-cell function from fasting plasma glucose and insulin concentrations in man. Diabetologia 28, 412-419.

31. Katz A, Nambi SS, Mather K, et al. (2000) Quantitative insulin sensitivity check index: a simple, accurate method for assessing insulin sensitivity in humans. J Clin Endocrinol Metab $\mathbf{8 5}$, 2402-2410.

32. Garmaroudi GR \& Moradi A (2010) Socio-economic status in Iran: a study of measurement index. PAYESH 9, 137-144.

33. Collier GR, Greenberg GR, Wolever TM, et al. (1988) The acute effect of fat on insulin secretion. J Clin Endocrinol Metab 66, 323-326.

34. Gannon MC, Nuttall FQ, Neil BJ, et al. (1988) The insulin and glucose responses to meals of glucose plus various proteins in type II diabetic subjects. Metab Clin Exp 37, 1081-1088.

35. Gannon MC, Nuttall FQ, Westphal SA, et al. (1993) The effect of fat and carbohydrate on plasma glucose, insulin, C-peptide, and triglycerides in normal male subjects. J Am Coll Nutr 12, 36-41.

36. Azadbakht L \& Esmaillzadeh A (2009) Soy-protein consumption and kidney-related biomarkers among type 2 diabetics: a crossover, randomized clinical trial. J Ren Nutr $\mathbf{1 9}$, 479-486.

37. Chaput J-P, Tremblay A, Rimm EB, et al. (2008) A novel interaction between dietary composition and insulin secretion: effects on weight gain in the Quebec Family Study. Am J Clin Nutr 87, 303-309.

38. McKeigue P, Shah B \& Marmot M (1991) Relation of central obesity and insulin resistance with high diabetes prevalence and cardiovascular risk in South Asians. Lancet 337 382-386.

39. Ludwig DS, Majzoub JA, Al-Zahrani A, et al. (1999) High glycemic index foods, overeating, and obesity. Pediatrics 103, E26.

40. Reaven GM (1988) Role of insulin resistance in human disease. Diabetes 37, 1595-1607.

41. Liu S, Manson JE, Stampfer MJ, et al. (2001) Dietary glycemic load assessed by food-frequency questionnaire in relation to plasma high-density-lipoprotein cholesterol and fasting plasma triacylglycerols in postmenopausal women. Am J Clin Nutr 73, 560-566.

42. Rock CL, Flatt SW, Thomson CA, et al. (2004) Plasma triacylglycerol and HDL cholesterol concentrations confirm selfreported changes in carbohydrate and fat intakes in women in a diet intervention trial. $J$ Nutr 134, 342-347.

43. De Rekeneire N, Peila R, Ding J, et al. (2006) Diabetes, hyperglycemia, and inflammation in older individuals: the Health, Aging and Body Composition Study. Diabetes Care 29, 1902-1908.

44. Esposito K, Marfella R \& Giugliano D (2003) Stress hyperglycemia, inflammation, and cardiovascular events. Diabetes Care 26, 1650-1651.

45. Steele RM, Brage S, Corder K, et al. (2008) Physical activity, cardiorespiratory fitness, and the metabolic syndrome in youth. $J$ Appl Physiol 105, 342-351.

46. Fedewa MV, Gist NH, Evans EM, et al. (2014) Exercise and insulin resistance in youth: a meta-analysis. Pediatrics 133, e163-e174.

47. Strawbridge RJ, Dupuis J, Prokopenko I, et al. (2011) Genomewide association identifies nine common variants associated with fasting proinsulin levels and provides new insights into the pathophysiology of type 2 diabetes. Diabetes $\mathbf{6 0}$, 2624-2634.

48. Manning AK, Hivert M-F, Scott RA, et al. (2012) A genomewide approach accounting for body mass index identifies genetic variants influencing fasting glycemic traits and insulin resistance. Nat Genet 44, 659-669.

49. Wang J, Wu Z, Li D, et al. (2012) Nutrition, epigenetics, and metabolic syndrome. Antioxid Redox Signaling 17, 282-301.

50. Alnasir FA \& Masuadi EM (2006) The effect of loss of body weight on lipid profile in overweight individuals. Saudi Med J 27, 687-692

51. Chang Y, Sung E, Yun KE, et al. (2013) Weight change as a predictor of incidence and remission of insulin resistance. PLOS ONE 8, e63690.

52. Vasunilashorn S (2013) Retrospective reports of weight change and inflammation in the US National Health and Nutrition Examination Survey. J Obes 2013, 601534. 